\title{
Antibody-mediated enzyme replacement therapy targeting both lysosomal and cytoplasmic glycogen in Pompe disease
}

\author{
Haiqing $\mathrm{Yi}^{1} \cdot$ Tao Sun $^{1} \cdot$ Dustin Armstrong ${ }^{2} \cdot$ Scott Borneman $^{3} \cdot$ Chunyu Yang $^{1}$ • \\ Stephanie Austin ${ }^{1} \cdot$ Priya S. Kishnani $^{1} \cdot$ Baodong Sun $^{1}$ (D)
}

Received: 27 September 2016/Revised: 13 December 2016 / Accepted: 2 January 2017 / Published online: 2 February 2017

(C) Springer-Verlag Berlin Heidelberg 2017

\begin{abstract}
Pompe disease is characterized by accumulation of both lysosomal and cytoplasmic glycogen primarily in skeletal and cardiac muscles. Mannose-6-phosphate receptor-mediated enzyme replacement therapy (ERT) with recombinant human acid $\alpha$ glucosidase (rhGAA) targets the enzyme to lysosomes and thus is unable to digest cytoplasmic glycogen. Studies have shown that anti-DNA antibody 3E10 penetrates living cells and delivers "cargo" proteins to the cytosol or nucleus via equilibrative nucleoside transporter ENT2. We speculate that 3E10-mediated ERT with GAA will target both lysosomal and cytoplasmic glycogen in Pompe disease. A fusion protein (FabGAA) containing a humanized Fab fragment derived from the murine 3E10 antibody and the $110 \mathrm{kDa}$ human GAA precursor was constructed and produced in $\mathrm{CHO}$ cells. Immunostaining with an anti-Fab antibody revealed that the Fab signals did not co-localize with the lysosomal marker LAMP2 in cultured L6 myoblasts or Pompe patient fibroblasts after incubation with FabGAA. Western blot with an anti-GAA antibody showed presence of the $150 \mathrm{kDa}$ full-length FabGAA in the cell lysates, in addition to the 95 - and $76 \mathrm{kDa}$ processed forms of GAA that were also seen in the rhGAA-treated cells. Blocking of mannose-6phosphate receptor with mannose-6-phosphate markedly reduced the 95 - and the $76 \mathrm{kDa}$ forms but not the $150 \mathrm{kDa}$ form. In GAA-KO mice, FabGAA achieved similar treatment
\end{abstract}

Haiqing Yi and Tao Sun contributed equally to this work.

Baodong Sun

baodong.sun@duke.edu

1 Division of Medical Genetics, Department of Pediatrics, Duke University School of Medicine, Durham, NC 27710, USA

2 Valerion Therapeutics, Concord, MA 01742, USA

3 Borneman Consulting, Del Mar, CA 92014, USA efficacy as rhGAA at an equal molar dose in reducing tissue glycogen contents. Our data suggest that FabGAA retains the ability of rhGAA to treat lysosomal glycogen accumulation and has the beneficial potential over rhGAA to reduce cytoplasmic glycogen storage in Pompe disease.

\section{Key messages}

- FabGAA can be delivered to both the cytoplasm and lysosomes in cultured cells.

- FabGAA equally reduced lysosomal glycogen accumulation as rhGAA in GAA-KO mice.

- FabGAA has the beneficial potential over rhGAA to clear cytoplasmic glycogen.

- This study suggests a novel antibody-enzyme fusion protein therapy for Pompe disease.

Keywords Pompe disease $\cdot 3$ E10 Fab $\cdot$ Enzyme replacement therapy · Recombinant human acid $\alpha$-glucosidase $\cdot$ Fusion protein $\cdot$ Cytoplasmic glycogen

\section{Introduction}

Pompe disease, also known as glycogen storage disease type II (GSD II, MIM 232300), is caused by a deficiency of the lysosomal enzyme acid $\alpha$-glucosidase (GAA; acid maltase; EC 3.2.1.20) that leads to lysosomal accumulation of glycogen in multiple tissues, with cardiac, skeletal, and smooth muscles being the most severely affected [1]. Infantile-onset Pompe disease is characterized by muscle weakness, hypotonia, and a hypertrophic cardiomyopathy, and most patients die from cardiorespiratory failure in the first year of life. Late-onset Pompe disease features progressive skeletal muscle weakness without significant cardiomyopathy [2-4]. Pompe disease is currently treated by enzyme replacement therapy (ERT) with recombinant human 
GAA (rhGAA, Alglucosidase alfa, Myozyme®) [5, 6]. ERT in Pompe disease is facilitated by cation-independent mannose-6phosphate receptor (M6PR)-mediated delivery of rhGAA to the lysosomes of target cells [7]. Although lysosomal glycogen accumulation has been considered a pathological hallmark of Pompe disease, increasing cytoplasmic glycogen accumulation resulting from rupture or shearing effect of lysosomes with disease progression occurs in both infantile- and late-onset Pompe disease, and exacerbates the damage of muscle cells [8-11]. As the delivery of rhGAA by ERT is limited to lysosomes through the M6PR-mediated endocytosis, a method that can extend the delivery of the therapeutic enzyme to cytosol is highly desired for Pompe disease.

The limited cytoplasmic delivery has been a major barrier to the development of protein drugs as clinical therapeutics. In the past decades, various approaches such as cell-penetrating peptide- or antibody-based drug delivery systems have been explored vigorously $[12,13]$. The monoclonal anti-DNA autoantibody $3 \mathrm{E} 10$, or its antigen-binding fragment $(\mathrm{Fab})$ or single-chain variable fragment ( $\mathrm{scFv}$ ), is able to penetrate cell membrane of living cells and can deliver a variety of proteins, including a large $305 \mathrm{kDa}$ alkaline phosphatase-conjugated goat antibody, to the cytosol or nuclei [14-16]. In addition, a recent study demonstrated that ERT with 3E10Fv-MTM1 fusion protein improved muscle function and pathology in mice with X-linked myotubular myopathy [17]. 3E10 penetrates cells via equilibrative nucleoside transporter 2 (ENT2) [18] that is expressed at high levels in human and rodent skeletal muscles and heart $[19,20]$, making it an ideal vehicle for cytoplasmic delivery of therapeutic GAA in muscle tissues of Pompe disease. A 3E10-GAA fusion should enable ENT2-mediated uptake to clear extra-lysosomal glycogen in muscle fibers while retaining the ability to degrade lysosomal glycogen via M6PR-mediated uptake.

Unlike the skeletal muscle of human patients with Pompe disease, muscles of GAA-KO mice do not have significant accumulation of cytoplasmic glycogen [21]. Yet GAA-KO mice remain a useful model of Pompe disease to examine processing of an antibody-hGAA fusion and to delineate whether the fusion protein retains the ability to clear lysosomal glycogen equally well compared to hGAA alone. Therefore, in this study, we examined the cell penetration and intracellular processing of the antibody-hGAA fusion (FabGAA) and determined its efficacy in enzyme replacement therapy in GAA-KO mice.

\section{Material and methods}

\section{FabGAA fusion protein and rhGAA}

A fusion protein (FabGAA) containing the humanized 3E10 Fab fragment and the $110 \mathrm{kDa}$ human GAA precursor
(Fig. 1a) was provided by Valerion Therapeutics (Concord, MA). Clinical grade Myozyme (rhGAA) was obtained from a physician whose patient with Pompe disease died during ERT. The two products had comparable specific activities per mole mass $\left(6.78 \times 10^{12} \mathrm{U} / \mathrm{mol}\right.$ for Myozyme vs $5.22 \times 10^{12} \mathrm{U} / \mathrm{mol}$ for FabGAA, measured by cleavage of artificial substrate 4methylumbelliferyl- $\alpha$-D-glucopyranoside as described below; $1 \mathrm{U}=1 \mathrm{nmol}$ per hour at $37^{\circ} \mathrm{C}$ and $\mathrm{pH} 4.3$ ).

\section{FabGAA uptake in cultured L6 myoblasts and GSD II patient fibroblast cells}

L6 rat myoblast and GSD II patient primary fibroblast cells were maintained in DMEM amended with $10 \%$ FBS in humidified $37^{\circ} \mathrm{C}, 5 \% \mathrm{CO}_{2}$ incubator. For FabGAA uptake assay, cells in a $10-\mathrm{cm}$ dish were incubated with $10-\mathrm{mL}$ medium plus $1000 \mathrm{U} /$ mL FabGAA for predetermined periods of time. Mannose-6phosphate was added as a competitive inhibitor of M6PR at a concentration of $5 \mathrm{mM}$ to the culture media where indicated. Cells were washed 4 times with cold PBS, scraped off plate, and pelleted at $800 \mathrm{~g}$ for $5 \mathrm{~min}$, then resuspended in $200-\mu \mathrm{L}$ cold deionized water, sonicated $15 \mathrm{~s} 3$ times with 10 -s intervals.

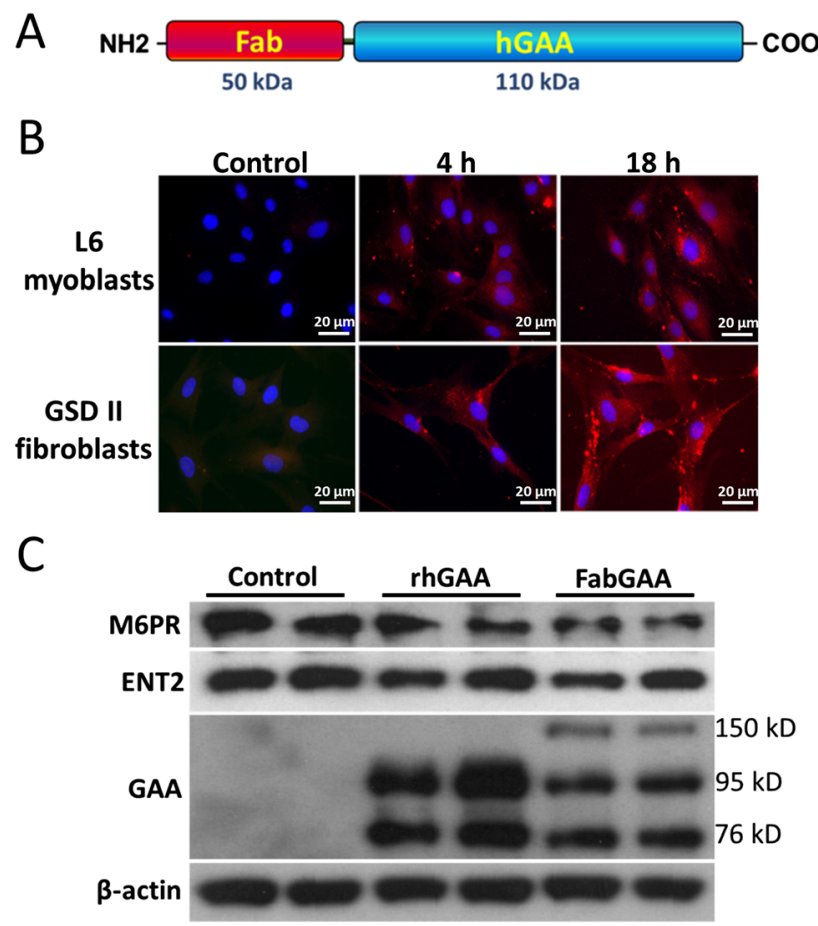

Fig. 1 Uptake of FabGAA by cultured cells. a A schematic diagram of the fusion FabGAA protein. b Immunostaining of Fab in L6 and GSD II (Pompe) patient primary fibroblast cells after incubating with $1000 \mathrm{U} / \mathrm{mL}$ of FabGAA for 4 or $18 \mathrm{~h}$. Alexa Fluor 594-conjugated anti-mouse secondary antibody that recognizes only the Fab fragment but not the hGAA fragment shows the presence of Fab in cells. Control, no FabGAA incubation. c Western blotting for expression of receptors in L6 cells and the presence of forms of GAA after incubation with $1000 \mathrm{U} / \mathrm{mL}$ of rhGAA or FabGAA for $18 \mathrm{~h}$ 
Lysates were cleared by centrifuging $10 \mathrm{~min}$ at $14,000 \mathrm{~g}$ and used for GAA activity assay (described below). For Western blotting, cell pellets were extracted in RIPA buffer and protein concentrations were measured with BCA method.

\section{Immunofluorescence}

Cells were grown on coverslips overnight and then incubated for indicated time in the presence or absence of $1000-\mathrm{U} / \mathrm{mL}$ FabGAA in humidified incubator at $37^{\circ} \mathrm{C}$ and $5 \% \mathrm{CO}_{2}$. Cells were then washed 4 times with cold PBS and fixed with $4 \%$ paraformaldehyde at room temperature for $15 \mathrm{~min}$. Immunofluorescence was done as described [22]. Briefly, fixed cells were permeabilized for 15 min with $0.1 \%$ Triton $\mathrm{X}-100$ and blocked with 5\% goat serum in DPBS for $30 \mathrm{~min}$, then incubated with rabbit anti-Lamp2 antibody (ab37024) (1:500 in blocking buffer) for $1 \mathrm{~h}$, then with Alexa Fluorconjugated secondary antibodies (Invitrogen) for $1 \mathrm{~h}$. Fab (of mouse origin) was visualized directly using an Alexa Fluor-conjugated anti-mouse secondary antibody (Invitrogen). Fluorescence images were taken with Leica DMI6000B microscope.

\section{Treatment of young adult GAA-KO mice with FabGAA}

Weekly ERT was conducted in GAA-KO mice [21] via tail vein injection of equal molar dose of FabGAA $(30 \mathrm{mg} / \mathrm{kg}$, $n=6)$ or untagged rhGAA ( $20 \mathrm{mg} / \mathrm{kg}, n=5)$ for 4 weeks starting from age of 12 weeks (note that the molar mass of FabGAA is approximately 1.5 times that of rhGAA); agematched untreated (UT, $n=6$ ) mice were kept as controls. Diphenhydramine $(15 \mathrm{mg} / \mathrm{kg})$ was intraperitoneally injected 10-15 min prior to each enzyme administration to prevent anaphylactic reactions [23].

\section{Treatment of old GAA-KO mice with FabGAA}

Low-dose treatment: Six 39 week-old GAA-KO mice were intravenously injected with $30 \mathrm{mg} / \mathrm{kg}$ FabGAA once per week for 8 weeks; seven UT mice were kept as controls.

High-dose treatment: Seven 49 week-old GAA-KO mice were intravenously injected with $90 \mathrm{mg} / \mathrm{kg}$ FabGAA once per week for 4 weeks; seven UT mice were kept as controls.

All mice included in this study were males. All animal procedures were done in accordance with Duke University Institutional Animal Care and Use Committee approved guidelines.

\section{Tissue sample collection}

Mice were sacrificed $48 \mathrm{~h}$ after the last injection following overnight fasting. For each mouse, urine was collected for testing urinary glucose tetrasaccharide Hex4, a biomarker of
Pompe disease [24]. A small portion of each tissue was fixed in $10 \%$ neutral buffered formalin for histology, and the rest was flash-frozen on dry ice and stored in $-80^{\circ} \mathrm{C}$ until use for biochemical analysis.

\section{Tissue glycogen staining}

Periodic acid-Schiff (PAS) staining was done at Duke Pathology Laboratory as described previously [25].

\section{Measurement of tissue glycogen contents, GAA activity, and urinary $\mathrm{Hex}_{4}$}

Frozen tissues were homogenized in cold water and centrifuged. The clear lysates were used for the following assays. GAA activity was assessed by a fluorogenic assay using the artificial substrate 4-methylumbelliferyl- $\alpha$-D-glucoside at $37{ }^{\circ} \mathrm{C}$ and $\mathrm{pH} 4.3$, and glycogen content was assayed by measuring the amount of glucose released from boiled tissue homogenate after digestion with amyloglucosidase from Aspergillus niger [26]. Protein concentration was measured using BCA method. Urinary Hex4 was measured by liquid chromatography-tandem mass spectrometry (LC-MS/MS) method [27].

\section{Western blotting}

Cells or tissues were homogenized in RIPA buffer and the clear lysates were subject to SDS-PAGE and Western blotting as previously described [28]. Primary antibodies used: mouse anti hGAA (home made from serum of GAA-knockout mice injected with rhGAA), rabbit anti ENT2 (Santa Cruz Biotechnology, sc-134569), mouse anti M6PR (Abcam, ab124767), mouse anti $\alpha$-tubulin (Sigma-Aldrich, T8203), and mouse anti $\beta$-actin (Sigma-Aldrich, A3854).

\section{Determination FabGAA's capability of degrading glycogen in vitro at neutral $\mathrm{pH}$}

The purpose of this experiment was to evaluate the glucosidase activity of FabGAA against glycogen in a cell-free setting at varying $\mathrm{pH}$. Glycogen solutions were prepared at a concentration of $10 \mathrm{mg} / \mathrm{mL}$ in citrate/phosphate buffers with $\mathrm{pH}$ values ranging from 3.5 to 7.0. At each $\mathrm{pH}$, triplicate reactions containing $178.2 \mu \mathrm{L}$ glycogen solution and $1.8 \mu \mathrm{L}$ of $1 \mathrm{mg} / \mathrm{mL}$ FabGAA ( $10 \mu \mathrm{g} / \mathrm{mL}$ final FabGAA concentration) were incubated at ambient temperature for $1 \mathrm{~h}$. Amounts of glucose generated in the reactions were determined using Glucose Oxidase kit (Sigma GAGO20-1KT). The glucosidase activity of FabGAA is expressed as nmol glucose released per minute per $\mathrm{mg}$ protein $(\mathrm{nmol} / \mathrm{min} / \mathrm{mg})$. 


\section{Statistical analysis}

Data were presented as mean \pm standard deviation. The significance of differences was assessed using two-tailed, equal variance Student's $t$ test. A $p$ value $<0.05$ was considered to be statistically significant.

\section{Results}

FabGAA can be delivered to both the cytoplasm and the lysosomes in cultured cells

To test whether Fab-tagged fusion protein is capable of being delivered into cells through both M6PR and ENT2, we incubated FabGAA with L6 myoblasts and primary fibroblasts from patients with Pompe disease. Immunostaining with an anti-Fab antibody, which recognizes only the Fab fragment but not human GAA, revealed increasing signal over time in both cells (Fig. 1b). Western blotting on L6 cell lysates using an anti-GAA antibody revealed that in addition to the typical 95 - and $76 \mathrm{kDa}$ processed (lysosomal) forms that are also seen in the rhGAA-treated cells [29], there existed a $150 \mathrm{kDa}$ fulllength FabGAA, presumably the cytoplasmic form, in the FabGAA-treated cells (Fig. 1c). When the same membrane was blotted with an anti-Fab antibody, only the $150 \mathrm{kDa}$ bands appeared in the FabGAA-treated cells (not shown). These data suggest that the Fab tag does not interfere with the maturation of GAA in lysosomes, and the tag is removed from the processed GAA forms (95 and $76 \mathrm{kDa})$. The majority of the absorbed FabGAA was destined to the lysosome via M6PR-mediated uptake.

To further confirm that the full-length FabGAA can be delivered through M6PR-independent pathway, we treated GSD II patient fibroblasts with FabGAA with or without the presence of M6P as a competitive inhibitor. Costaining of Fab and the lysosomal marker LAMP2 revealed that the two had little co-localization (Fig. 2a), indicating that the majority of the full-length FabGAA in cells is outside the lysosomes. Blocking of M6PR by M6P did not affect the Fab signal (Fig. 2a) but markedly reduced GAA activity in the FabGAA-treated cells (Fig. 2b). Furthermore, M6P significantly reduced the amount of the lysosomal forms of GAA (95 and $76 \mathrm{kDa}$ ), but did not affect the full-length form $(150 \mathrm{kDa})$ as shown by Western blotting (Fig. 2c). Similar phenomena were observed in L6 cells (not shown). These indicate that the fusion protein can be transported into the cells through M6PR and also through an additional route, presumably ENT2. The Fab fragment seemed to be quickly degraded from FabGAA once in the lysosome, as very little co-staining of Fab and LAMP2 could be observed (Fig. 2a).
FabGAA reduced glycogen accumulation in tissues of young adult GAA-KO mice

The ability of FabGAA to reverse the glycogen storage in GAA-KO mice was examined by intravenously administering the fusion protein to young adult GAA-KO mice (12 weeks old) in a 4 week enzyme replacement protocol, in comparison with rhGAA treatment. Mice received weekly injection of equal molar dose of rhGAA $(20 \mathrm{mg} / \mathrm{kg})$ or FabGAA (30 mg/kg). Both treatments resulted in very high GAA activities in liver and moderately elevated GAA activities in heart and skeletal muscles measured $48 \mathrm{~h}$ after the fourth injection (Fig. 3a). The two treatments achieved comparable reduction of glycogen content in major affected tissues (Fig. 3b). Reduction of glycogen storage in tissues of GAA-KO mice by FabGAA treatment was also evidenced by PAS staining (Fig. 3c). The results suggest that FabGAA can be delivered into lysosomes and can degrade lysosomal glycogen in GAA-KO mice as efficiently as rhGAA. Urinary $\mathrm{Hex}_{4}$ was significantly reduced in both the rhGAA- and FabGAA-treated mice (Fig. 3d), indicating similar alleviation of the disease by both treatments.

\section{Distribution of FabGAA in mouse tissues was heterogeneous}

We examined the distribution of rhGAA and FabGAA in tissues of these treated mice. Liver had the highest protein levels for both enzymes (Fig. 3e), which coincides with the fact that liver has the most abundant expression of M6PR that facilitates transportation of rhGAA or FabGAA to the lysosomes [30]. Quadriceps had the lowest levels for both rhGAA and FabGAA, which is consistent with the previously observed low efficacy of ERT in skeletal muscles [30]. Both rhGAA and FabGAA proteins presented predominately as the mature lysosomal forms (76 and $67 \mathrm{kDa}$ ) in all tissues, indicating that both proteins were destined to the lysosomes (Fig. 3e). Surprisingly, no cytoplasmic $150 \mathrm{kDa}$ fulllength FabGAA was detected in any tissues of the FabGAA-treated mice (Fig. 3e). We suspected that the protein was not stable in the cytoplasm and was degraded within $48 \mathrm{~h}$ after the enzyme injection.

To investigate whether ENT2-mediated FabGAA uptake occurs in vivo in mouse tissues, we conducted a short-term FabGAA treatment: GAA-KO mice were intravenously injected with FabGAA $(30 \mathrm{mg} / \mathrm{kg})$ and tissues were harvested 3 and $9 \mathrm{~h}$ later after perfusion with $25-\mathrm{mL}$ PBS to eliminate blood contamination. At $3 \mathrm{~h}$ after injection, the amount of the full-length FabGAA protein $(150 \mathrm{kDa})$ accounted for roughly one half of the total protein detected in both the cardiac and skeletal muscles, and the uptake efficiency in heart is apparently higher than in skeletal muscles (Fig. 3f). Little $150 \mathrm{kDa}$ FabGAA was detected in liver where the protein existed 
Fig. 2 Uptake of FabGAA by fibroblasts from Pompe patient was through M6PR-dependent and independent pathways. a FabGAA (green) and the lysosomal marker LAMP2 (red) do not co-localize in cells that were incubated with $1000 \mathrm{U} / \mathrm{mL}$ FabGAA for $18 \mathrm{~h}$; addition of M6P to the medium does not abolish the signal of FabGAA. b GAA activity in cell lysates after $18 \mathrm{~h}$ incubation with $1000 \mathrm{U} / \mathrm{mL}$ FabGAA, with or without M6P; data are shown as mean $\pm \mathrm{SD}$. c Cells treated with $1000 \mathrm{U} / \mathrm{mL}$ FabGAA for $18 \mathrm{~h}$ show the presence of full-length FabGAA $(150 \mathrm{kDa})$ and its processed lysosomal forms (95 and $76 \mathrm{kDa}$ ) in immunoblot. Addition of M6P to the medium does not affect the $150 \mathrm{kDa}$ form but greatly reduced the amount of the lysosomal forms

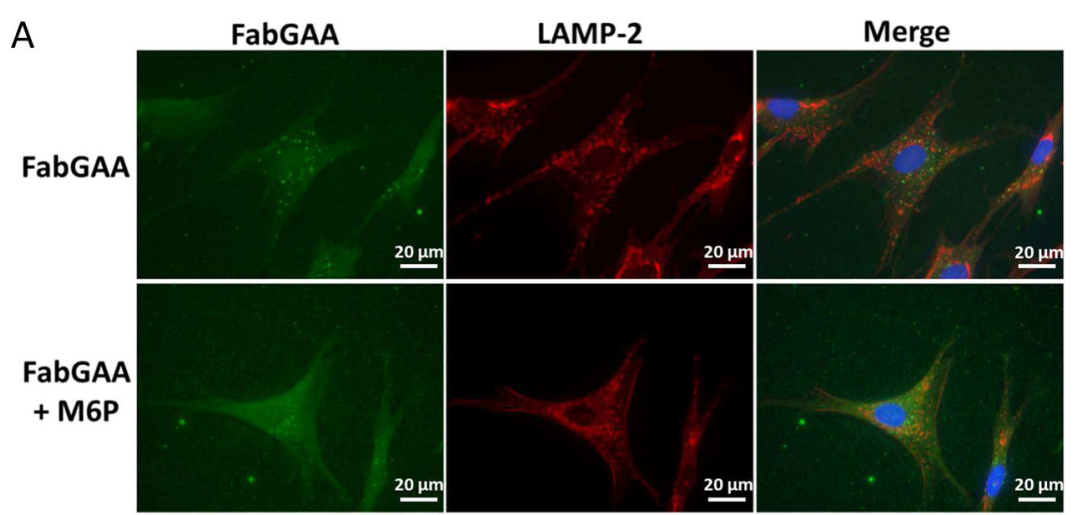

B

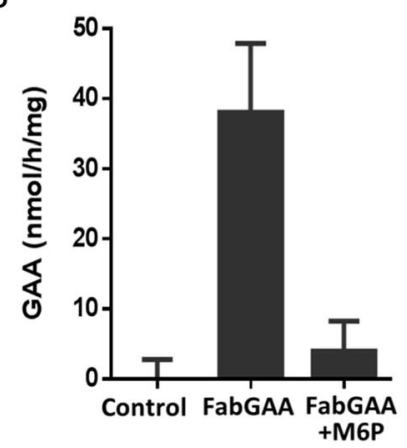

C

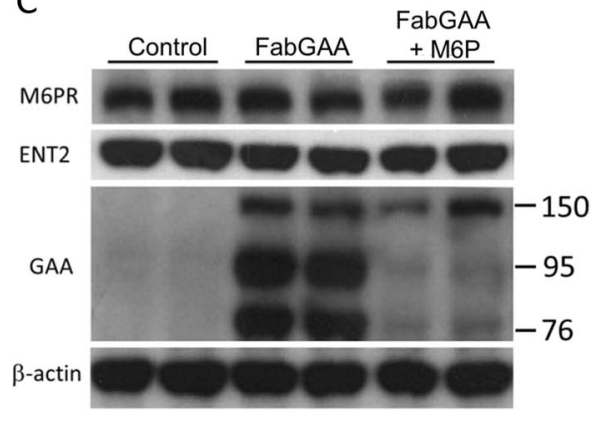

predominately as the $76 \mathrm{kDa}$ lysosomal form (Fig. 3f), which is consistent with low expression of ENT2 and high expression of M6PR in this tissue [20,30]. At $9 \mathrm{~h}$, the amount of fulllength FabGAA was significantly reduced while the $76 \mathrm{kDa}$ form remained unchanged in heart and skeletal muscles (Fig. 3f), suggesting that the full-length protein was quickly degraded in the cytoplasm.

\section{Correction of glycogen storage in old GAA-KO mice requires high dose of FabGAA}

Progressive glycogen accumulation in muscle tissues in GAA$\mathrm{KO}$ mice leads to development of significant muscle wasting at advanced ages [21]. Animal studies have shown that the outcome of treating old GAA-KO mice is much less effective than treating young mice $[31,32]$. To test the efficacy of FabGAA treatment in GAA-KO mice at advanced ages, we performed a low-dose $(30 \mathrm{mg} / \mathrm{kg}), 8$ week treatment starting at age of 39 weeks, and a high-dose $(90 \mathrm{mg} / \mathrm{kg}), 4$ week treatment starting at age 49 weeks. As shown in Fig. 4, the low-dose treatment achieved tissue GAA activity elevation to magnitudes comparable with those seen in young GAA-KO mice treated for 4 weeks with the same dose of FabGAA (Fig. 3a), but moderate glycogen reduction was observed only in liver $(-51 \%)$ and heart $(-10 \%)$ by the treatment. In contrast, the high-dose treatment resulted in dramatically higher GAA activity in all tissues (Fig. 5a), and consequently, glycogen content was reduced by $47 \%$ in liver, $36 \%$ in heart, $25 \%$ in quadriceps, $36 \%$ in gastrocnemius, and 39\% in diaphragm (Fig. 5b); these results were supported by PAS staining of these tissues (Fig. 5c).

\section{FabGAA retains significant capability of degrading glycogen at neutral $\mathrm{pH}$}

Due to the absence of cytoplasmic glycogen accumulation in muscles of the GAA-KO mouse model, direct assessment of FabGAA's ability to digest cytoplasmic glycogen was not possible, thus we assessed the ability of FabGAA to digest glycogen at various $\mathrm{pH}$ in vitro. Excessive amount of glycogen was dissolved in buffers with $\mathrm{pH}$ values ranging from 3.5 to 7.0 and incubated with FabGAA at ambient temperature for $1 \mathrm{~h}$. The amounts of glucose generated in the reactions were expressed as glucosidase activity of FabGAA. As shown in Fig. 6, the activity of FabGAA at $\mathrm{pH} 7.0$ (cytosolic $\mathrm{pH}$ ) was approximately $18 \%$ of that at $\mathrm{pH} 4.5$ (lysosomal $\mathrm{pH}$ ). This suggests that FabGAA retains significant glycogen hydrolysis activity in the cytoplasm. Given large enough amount, the fusion protein has the capability of degrading cytoplasmic glycogen.

\section{Discussion}

Pompe disease is the only glycogen storage disease that is also classified as a lysosomal storage disease. Progressive accumulation of lysosomal glycogen has been considered as the major cause of cardiac and skeletal myopathy in patients with Pompe 


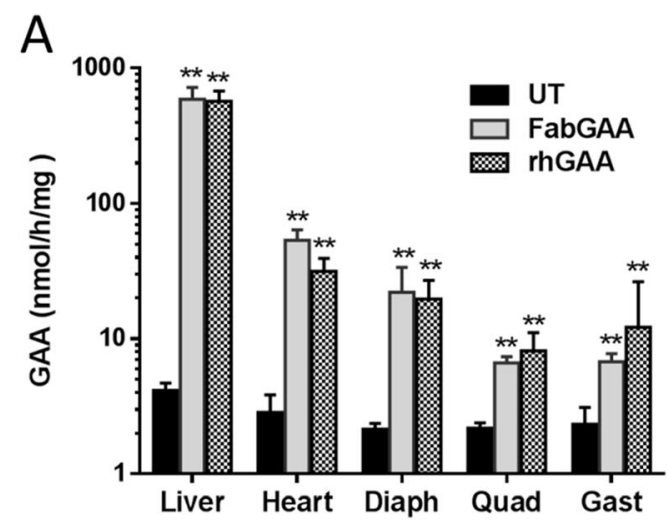

C

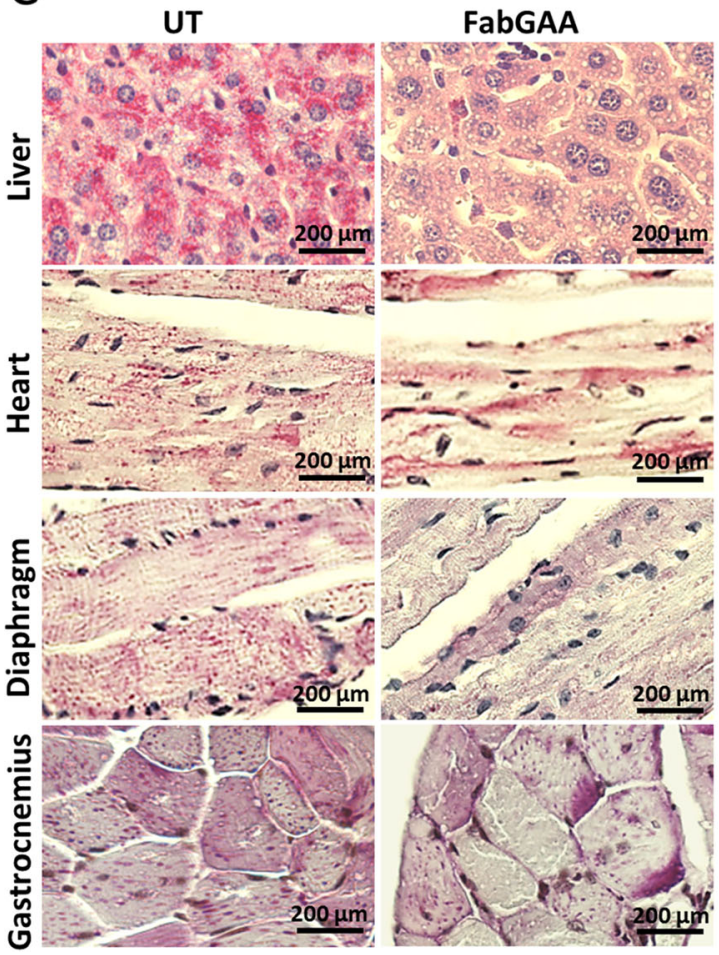

Fig. 3 FabGAA treatment elevated GAA activity and reduced glycogen levels in GAA-KO mouse tissues. a Weekly intravenous injection of 30 $\mathrm{mg} / \mathrm{kg}$ FabGAA or $20 \mathrm{mg} / \mathrm{kg}$ rhGAA into GAA-KO mice for 4 weeks resulted in high GAA activity in liver and moderately elevated activities in other tissues. b FabGAA treatment reduced glycogen content by $64 \%$ in liver, $55 \%$ in heart, $40 \%$ in diaphragm, $15 \%$ in quadriceps, and $38 \%$ in gastrocnemius. The efficacy of FabGAA was similar to rhGAA in all tissues. UT untreated. c PAS staining showing reduction of glycogen accumulation by FabGAA treatment in major affected tissues. d

disease. However, the impact of increasing cytoplasmic glycogen accumulation with disease progression on Pompe pathology is often overlooked. Electron microscopic observations of massive cytoplasmic glycogen accumulation, in addition to glycogen stored in the lysosomes, have been frequently reported in skeletal muscles in both infantile and late-onset Pompe patients [8-10]. A lysosomal rupture hypothesis was proposed by Griffin as the source of cytoplasmic accumulation in muscles of Pompe disease [11]. This hypothesis suggests that, with
B

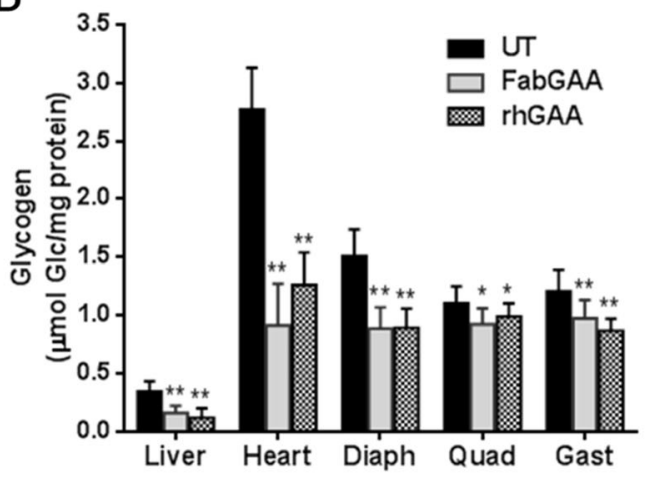

D
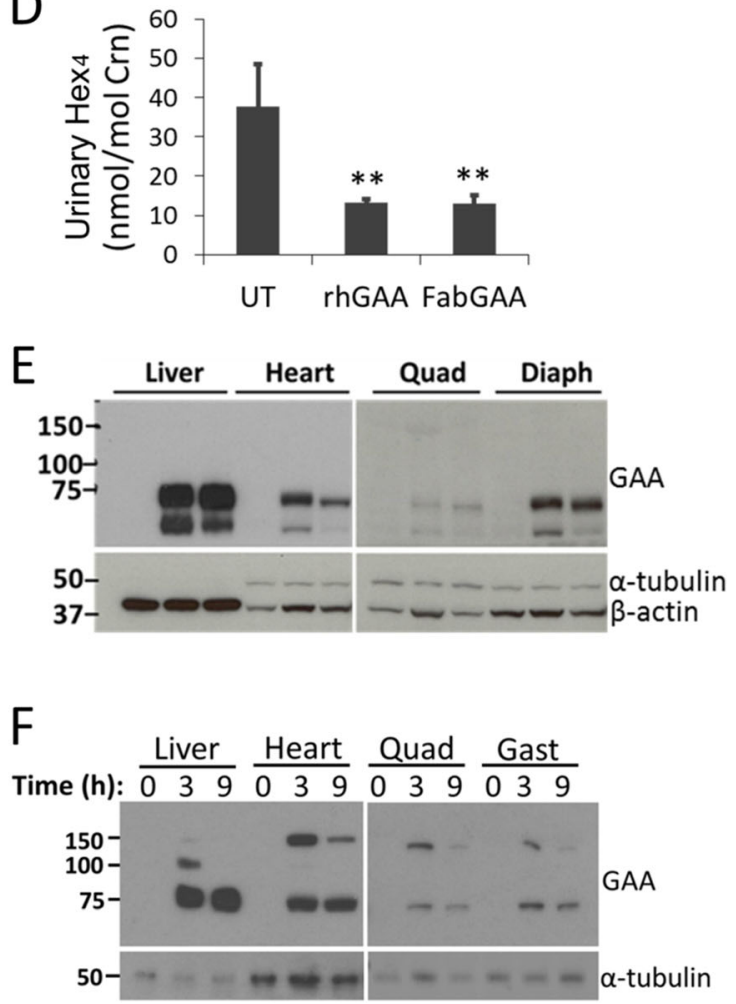

Urinary $\mathrm{Hex}_{4}$ concentration after 4 week treatment with rhGAA or FabGAA. e Representative immunoblots showing GAA in tissues after 4 week treatment with rhGAA or FabGAA. The loading order for each tissue is untreated, rhGAA treated, and FabGAA treated. Four microgram total protein was loaded for liver, $20 \mu \mathrm{g}$ for all other tissues. $\mathbf{f}$ Detection of FabGAA in tissues 3 and $9 \mathrm{~h}$ after injection of $30 \mathrm{mg} / \mathrm{kg}$ FabGAA. Fulllength protein is $150 \mathrm{kDa}$; the mature form is $76 \mathrm{kDa}$. In $\mathbf{a}, \mathbf{b}$, and $\mathbf{d}, n=6$ for all groups; data are shown as mean $\pm \mathrm{SD} ; * p<0.05$ and $* * p<0.01$, comparing with UT

disease progression, movement and increased myofibril rigidity during contraction cause enlarged lysosomes to rupture and release glycogen and lytic enzymes into the cytosol, which subsequently cause damage to the structure of muscle cells [11]. This may explain why muscle fibers are destroyed, while other cells like hepatocytes or macrophages that mostly accumulate glycogen in lysosomes are less affected in Pompe disease. Histopathologic studies of muscle biopsies from infantileonset Pompe patients reveal that early stage muscle cells have 
Fig. 4 GAA activity and glycogen contents in tissues of old GSD II mice treated with lowdose FabGAA. Mice were weekly injected with $30 \mathrm{mg} / \mathrm{kg}$ FabGAA for 8 weeks starting from age of 39 weeks. $n=7$ for UT, $n=6$ for FabGAA treated; data are shown as mean $\pm \mathrm{SD} ; * p<0.05$, $* * p<0.01$
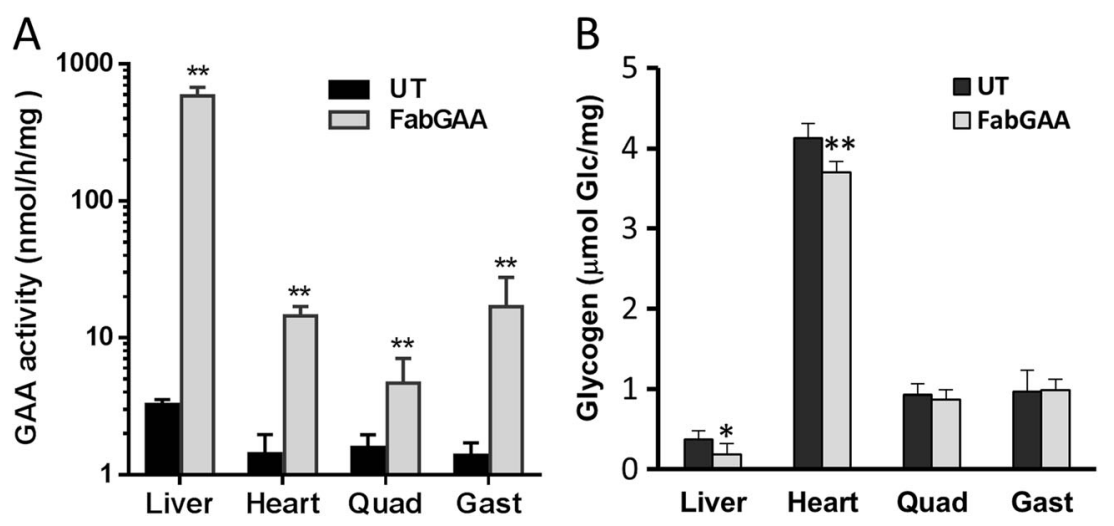

predominantly membrane-bound lysosomal glycogen; intermediate stage cells exhibit significant rupture of lysosomal membranes and mixture of free cytoplasmic glycogen and glycogen tightly packed in enlarged lysosomes; at late stages, contractile elements of myocytes are replaced by pooling of cytoplasmic glycogen, which eventually lead to destruction of muscle fibers $[9,11]$.

M6PR is the major cell-surface receptor through which therapeutic rhGAA is delivered from the circulating system to lysosomes in cells during ERT of Pompe patients [30]. Patients with early stage disease respond well to ERT but the treatment outcome is poor for the patients in later stages of disease [9]. This suggests that a new treatment approach that can target both lysosomal and cytoplasmic glycogen is required for Pompe disease.
The poor cytoplasmic delivery has been a major barrier to the development of protein drugs as clinical therapeutics. Numerous approaches such as peptide-modified drug delivery systems have been explored in the past decades [12, 13]. One extensively studied method is to modify the protein with a cell-penetrating peptide (CPP). CPPs, also known as proteintransduction domains, are short, basic peptides that are capable of entering cells without the need of a specific receptor [33]. Fusion of CPPs, such as HIV-1 Tat peptide or HSV VP22 peptide, to other proteins has been used to deliver a variety of proteins to living cells [34]. Although CPPs represent an emerging tool for protein drug delivery, some disadvantages exist including susceptibility to cleavage by plasma enzymes, low efficiency to deliver large cargos, and likelihood of causing severe toxicity to kidney and liver [35].
Fig. 5 High-dose FabGAA treatment elevated GAA activity and reduced glycogen levels in tissues of old GAA-KO mice. a Weekly injection of FabGAA at $90 \mathrm{mg} / \mathrm{kg}$ for 4 weeks resulted in significantly higher GAA activity, especially in liver and heart. b High-dose FabGAA treatment reduced glycogen content by $47 \%$ in liver, $36 \%$ in heart, $25 \%$ in quadriceps, $36 \%$ in gastrocnemius, and $39 \%$ in diaphragm. $n=7$ for both FabGAA treated and UT; data are shown as mean $\pm \mathrm{SD} ; * * p<0.01$. c Representative PAS staining of major affected tissues.
A

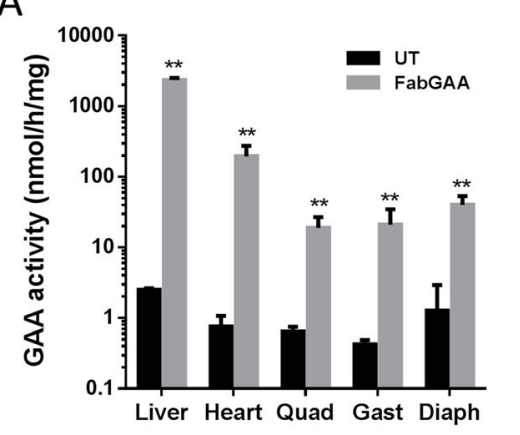

C

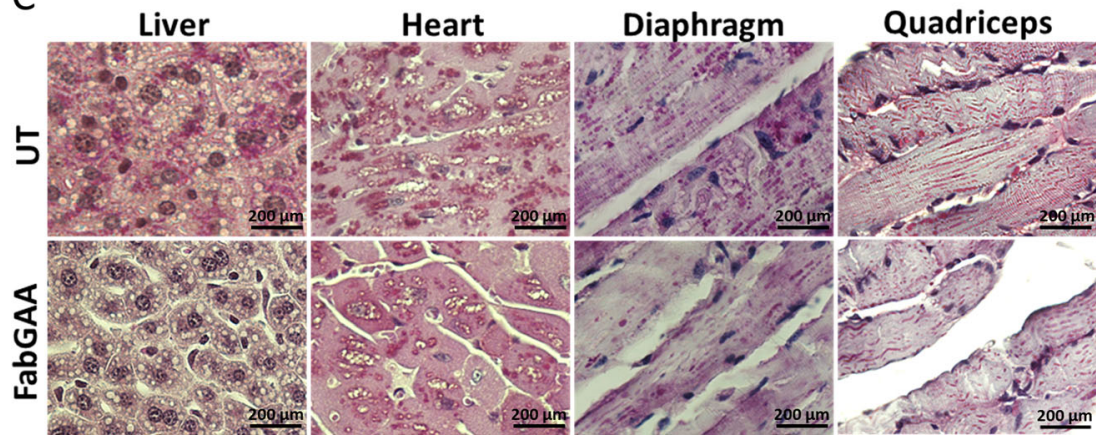

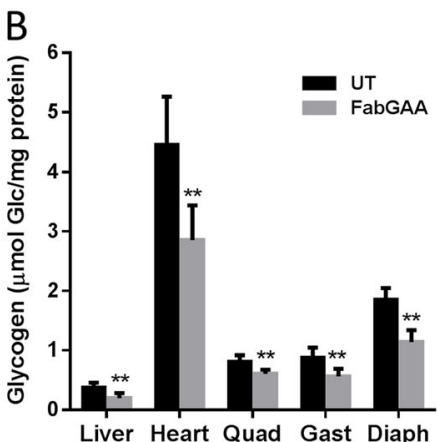

Quadriceps

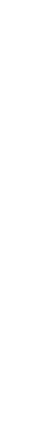




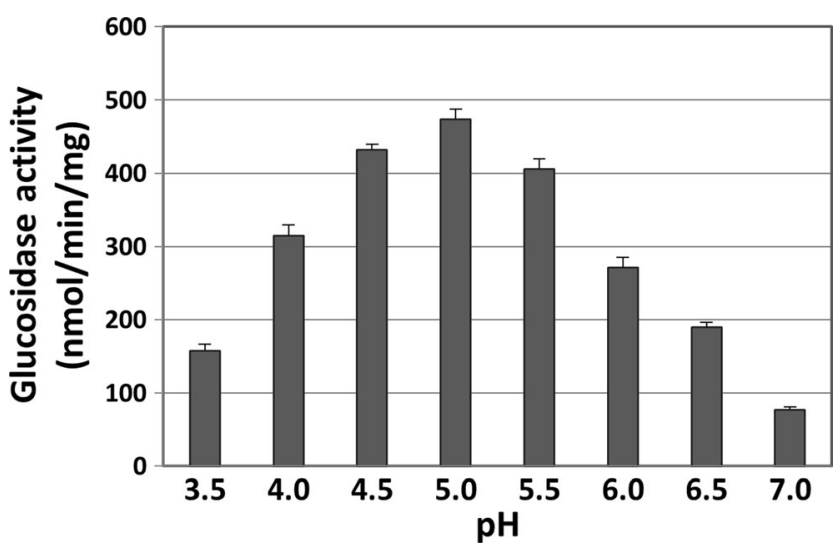

Fig. 6 The ability of FabGAA to degrade glycogen in vitro at various $\mathrm{pH}$. Glycogen solutions were prepared in buffers with varying $\mathrm{pH}$ values and digested by FabGAA (10 $\mu \mathrm{g} / \mathrm{mL}$ final concentration) at ambient temperature for $1 \mathrm{~h}$. The amounts of glucose generated in the reactions were expressed as glucosidase activity of FabGAA (nmol glucose released per minute per $\mathrm{mg}$ protein, $\mathrm{nmol} / \mathrm{min} / \mathrm{mg}$ ). Mean $\pm \mathrm{SD}$ are shown; $n=3$ for each $\mathrm{pH}$ value

The unique monoclonal antibody 3E10 and its fragments are capable of penetrating living cells to deliver functional proteins to the cytosol and nuclei via ENT2, a cell-surface transporter that is highly expressed in human skeletal and cardiac muscle $[18,19]$. More impressively, its Fab fragment has been shown to be able to deliver a $305 \mathrm{kDa}$ protein efficiently into cultured cells [16]. Addition of the 3E10 Fab tag to the GAA molecule (FabGAA fusion) provides an ideal system to permit the delivery of GAA to both lysosomes and the cytoplasm in target tissues of Pompe disease through two distinct pathways. In this study, we demonstrated that intravenously administration of FabGAA to GAA-KO mice resulted in similar levels of protein delivery to the cytoplasm and to the lysosomes, both in heart and skeletal muscles (Fig. 3f). In lysosomes, the FabGAA was similarly processed as rhGAA into mature forms; in the cytoplasm, FabGAA seemed to be unstable and was quickly degraded (Fig. 3f). Although FabGAA treatment reached comparable efficacy to rhGAA in reducing lysosomal glycogen accumulation in GAA-KO mice (Fig. 3a-e), its ability to clear cytoplasmic glycogen storage, which is absent in this animal model, could not be directly assessed in this study.

Though GAA is a native lysosomal enzyme, unprocessed FabGAA fusion protein was demonstrated to retain significant glucosidase activity against glycogen across a wide $\mathrm{pH}$ range in our in vitro assay (Fig. 6). The enzyme activity of FabGAA at $\mathrm{pH} 7.0$ (cytosolic $\mathrm{pH}$ ) was approximately $18 \%$ of that at $\mathrm{pH}$ 4.5 (lysosomal pH). A reduction in enzyme activity combined with reduced stability at neutral $\mathrm{pH}$ may necessitate more frequent administration and/or higher doses of FabGAA to achieve therapeutic efficacy in the cytoplasm.

Taken together, our data suggest that FabGAA retains the ability of rhGAA to treat lysosomal glycogen accumulation and has the beneficial potential over rhGAA to reduce cytoplasmic glycogen storage in Pompe disease. It could also possibly be used for treatment of other cytoplasmic glycogen storage diseases. Valerion Therapeutics intends to initiate clinical trials with this FabGAA fusion protein (VAL-1221) in patients with Pompe disease in early 2017.

Acknowledgements This work was supported by a grant from Valerion Therapeutics (Concord, MA). We thank Dr. Tracy R. McKnight for reviewing and editing the manuscript. We also thank Ms. Fengqin Gao for her assistance with animal care.

\section{Compliance with ethical standards}

Conflict of interest D. Armstrong is the founder of Valerion Therapeutics and declares ownership interest in the company. All other authors declare no competing or financial interests.

\section{References}

1. van der Ploeg AT, Reuser AJ (2008) Pompe's disease. Lancet 372(9646):1342-1353

2. Kishnani PS, Hwu WL, Mandel H, Nicolino M, Yong F, Corzo D (2006) A retrospective, multinational, multicenter study on the natural history of infantile-onset Pompe disease. J Pediatr 148(5):671676

3. Muller-Felber W, Horvath R, Gempel K, Podskarbi T, Shin Y, Pongratz D, Walter MC, Baethmann M, Schlotter-Weigel B, Lochmuller H et al (2007) Late onset Pompe disease: clinical and neurophysiological spectrum of 38 patients including long-term follow-up in 18 patients. Neuromuscul Disord 17(9-10):698-706

4. Kishnani PS, Beckemeyer AA, Mendelsohn NJ (2012) The new era of Pompe disease: advances in the detection, understanding of the phenotypic spectrum, pathophysiology, and management. Am J Med Genet C Semin Med Genet 160(1):1-7

5. Angelini C, Semplicini C (2012) Enzyme replacement therapy for Pompe disease. Curr Neurol Neurosci Rep 12(1):70-75

6. Case LE, Beckemeyer AA, Kishnani PS (2012) Infantile Pompe disease on ERT: update on clinical presentation, musculoskeletal management, and exercise considerations. Am J Med Genet C Semin Med Genet 160(1):69-79

7. Van Hove JL, Yang HW, Wu JY, Brady RO, Chen YT (1996) Highlevel production of recombinant human lysosomal acid alphaglucosidase in Chinese hamster ovary cells which targets to heart muscle and corrects glycogen accumulation in fibroblasts from patients with Pompe disease. Proc Natl Acad Sci U S A 93(1):65-70

8. Garancis JC (1968) Type II glycogenosis. Biochemical and electron microscopic study. Am J Med 44(2):289-300

9. Thurberg BL, Lynch Maloney C, Vaccaro C, Afonso K, Tsai AC, Bossen E, Kishnani PS, O'Callaghan M (2006) Characterization of pre- and post-treatment pathology after enzyme replacement therapy for Pompe disease. Lab Invest 86(12):1208-1220

10. Lewandowska E, Wierzba-Bobrowicz T, Rola R, Modzelewska J, Stepien T, Lugowska A, Pasennik E, Ryglewicz D (2008) Pathology of skeletal muscle cells in adult-onset glycogenosis type II (Pompe disease): ultrastructural study. Folia Neuropathol 46(2): 123-133

11. Griffin JL (1984) Infantile acid maltase deficiency. I. Muscle fiber destruction after lysosomal rupture. Virchows Arch B Cell Pathol Incl Mol Pathol 45(1):23-36 
12. Rehman K, Hamid Akash MS, Akhtar B, Tariq M, Mahmood A, Ibrahim M (2016) Delivery of therapeutic proteins: challenges and strategies. Curr Drug Targets 17(10):1172-1188

13. Komin A, Russell LM, Hristova KA, Searson PC (2016) Peptidebased strategies for enhanced cell uptake, transcellular transport, and circulation: mechanisms and challenges. Adv Drug Deliv Rev. doi:10.1016/j.addr.2016.06.002

14. Zack DJ, Stempniak M, Wong AL, Taylor C, Weisbart RH (1996) Mechanisms of cellular penetration and nuclear localization of an anti-double strand DNA autoantibody. J Immunol 157(5):20822088

15. Hansen JE, Sohn W, Kim C, Chang SS, Huang NC, Santos DG, Chan G, Weisbart RH, Nishimura RN (2006) Antibody-mediated Hsp70 protein therapy. Brain Res 1088(1):187-196

16. Weisbart RH, Stempniak M, Harris S, Zack DJ, Ferreri K (1998) An autoantibody is modified for use as a delivery system to target the cell nucleus: therapeutic implications. J Autoimmun 11(5):539-546

17. Lawlor MW, Armstrong D, Viola MG, Widrick JJ, Meng H, Grange RW, Childers MK, Hsu CP, O'Callaghan M, Pierson CR et al (2013) Enzyme replacement therapy rescues weakness and improves muscle pathology in mice with X-linked myotubular myopathy. Hum Mol Genet 22(8):1525-1538

18. Hansen JE, Tse CM, Chan G, Heinze ER, Nishimura RN, Weisbart RH (2007) Intranuclear protein transduction through a nucleoside salvage pathway. J Biol Chem 282(29):20790-20793

19. Pennycooke M, Chaudary N, Shuralyova I, Zhang Y, Coe IR (2001) Differential expression of human nucleoside transporters in normal and tumor tissue. Biochem Biophys Res Commun 280(3):951-959

20. Lu H, Chen C, Klaassen C (2004) Tissue distribution of concentrative and equilibrative nucleoside transporters in male and female rats and mice. Drug Metab Dispos 32(12):1455-1461

21. Raben N, Nagaraju K, Lee E, Kessler P, Byrne B, Lee L, LaMarca M, King C, Ward J, Sauer B et al (1998) Targeted disruption of the acid alpha-glucosidase gene in mice causes an illness with critical features of both infantile and adult human glycogen storage disease type II. J Biol Chem 273(30):19086-19092

22. Maga JA, Zhou J, Kambampati R, Peng S, Wang X, Bohnsack RN, Thomm A, Golata S, Tom P, Dahms NM et al (2013) Glycosylation-independent lysosomal targeting of acid alphaglucosidase enhances muscle glycogen clearance in Pompe mice. J Biol Chem 288(3):1428-1438

23. Joseph A, Munroe K, Housman M, Garman R, Richards S (2008) Immune tolerance induction to enzyme-replacement therapy by coadministration of short-term, low-dose methotrexate in a murine Pompe disease model. Clin Exp Immunol 152(1):138-146
24. Hallgren P, Hansson G, Henriksson KG, Hager A, Lundblad A, Svensson S (1974) Increased excretion of a glucose-containing tetrasaccharide in the urine of a patient with glycogen storage disease type II (Pompe's disease). Eur J Clin Invest 4(6):429-433

25. Yi H, Thurberg BL, Curtis S, Austin S, Fyfe J, Koeberl DD, Kishnani PS, Sun B (2012) Characterization of a canine model of glycogen storage disease type IIIa. Dis Model Mech 5(6):804-811

26. Amalfitano A, McVie-Wylie AJ, Hu H, Dawson TL, Raben N, Plotz P, Chen YT (1999) Systemic correction of the muscle disorder glycogen storage disease type II after hepatic targeting of a modified adenovirus vector encoding human acid-alpha-glucosidase. Proc Natl Acad Sci U S A 96(16):8861-8866

27. Young SP, Stevens RD, An Y, Chen YT, Millington DS (2003) Analysis of a glucose tetrasaccharide elevated in Pompe disease by stable isotope dilution-electrospray ionization tandem mass spectrometry. Anal Biochem 316(2):175-180

28. Yi H, Fredrickson KB, Das S, Kishnani PS, Sun B (2013) Stbd1 is highly elevated in skeletal muscle of Pompe disease mice but suppression of its expression does not affect lysosomal glycogen accumulation. Mol Genet Metab 109(3):312-314

29. Moreland RJ, Jin X, Zhang XK, Decker RW, Albee KL, Lee KL, Cauthron RD, Brewer K, Edmunds T, Canfield WM (2005) Lysosomal acid alpha-glucosidase consists of four different peptides processed from a single chain precursor. J Biol Chem 280(8):6780-6791

30. Raben N, Danon M, Gilbert AL, Dwivedi S, Collins B, Thurberg BL, Mattaliano RJ, Nagaraju K, Plotz PH (2003) Enzyme replacement therapy in the mouse model of Pompe disease. Mol Genet Metab 80(1-2):159-169

31. Raben N, Jatkar T, Lee A, Lu N, Dwivedi S, Nagaraju K, Plotz PH (2002) Glycogen stored in skeletal but not in cardiac muscle in acid alpha-glucosidase mutant (Pompe) mice is highly resistant to transgene-encoded human enzyme. Mol Ther 6(5):601-608

32. Sun B, Zhang H, Bird A, Li S, Young SP, Koeberl DD (2009) Impaired clearance of accumulated lysosomal glycogen in advanced Pompe disease despite high-level vector-mediated transgene expression. J Gene Med 11(10):913-920

33. Stewart KM, Horton KL, Kelley SO (2008) Cell-penetrating peptides as delivery vehicles for biology and medicine. Org Biomol Chem 6(13):2242-2255

34. Skotland T, Iversen TG, Torgersen ML, Sandvig K (2015) Cellpenetrating peptides: possibilities and challenges for drug delivery in vitro and in vivo. Molecules 20(7):13313-13323

35. Sarko D, Beijer B, Garcia Boy R, Nothelfer EM, Leotta K, Eisenhut M, Altmann A, Haberkorn U, Mier W (2010) The pharmacokinetics of cell-penetrating peptides. Mol Pharm 7(6):2224-2231 